\title{
Prevalência do uso do preservativo por trabalhadores da construção civil e fatores associados
}

\author{
Prevalence of condom use among construction workers and associated factors \\ Prevalencia del uso de condón por los trabajadores de la construcción y factores relacionados
}

\author{
Layane Trindade de Souza', Maria Aparecida Cavalcanti Catão", Maria Eliane Moreira Freire"II, \\ Wynne Pereira Nogueira ${ }^{\prime \prime}$, Cibelly Nunes Fortunato ${ }^{v}$, Ana Cristina de Oliveira e Silva ${ }^{v 1}$
}

\begin{abstract}
RESUMO
Objetivo: estimar a prevalência do uso do preservativo e os fatores associados em trabalhadores da construção civil. Método: estudo transversal, analítico, com 370 trabalhadores da grande João Pessoa, Paraíba. Considerou como variável de desfecho o uso do preservativo na última relação sexual. Utilizou-se questionário estruturado. Análise de regressão logística bivariada e múltipla foi utilizada para identificar associação entre as variáveis sociodemográficas e o uso do preservativo. Resultados: a maioria dos participantes é do sexo masculino, adultos jovens, casados e com baixa escolaridade. A prevalência estimada de uso do preservativo foi de $23,5 \%$ (IC 95\%: 19,2\% - 27,8\%). Indivíduos com menos de 39 anos possuem 1,82 vezes mais chances de usar o preservativo e ser casado diminui $(\mathrm{RC}=0,26)$ as chances de uso. Conclusão: há baixa prevalência de uso do preservativo em trabalhadores da construção civil. A prevenção combinada é uma alternativa para controle das infecções transmissíveis, sendo o preservativo o principal coadjuvante.
\end{abstract}

Descritores: Indústria da Construção; Saúde do Trabalhador; Preservativos; Prevalência; Enfermagem.

\section{ABSTRACT}

Objective: to estimate the prevalence of condom use and the associated factors in construction workers. Method: in this crosssectional, analytical study with 370 workers from greater João Pessoa, Paraíba, Brazil, the outcome variable was condom use at last sexual intercourse. A structured questionnaire was used. Bivariate and multiple logistic regression analysis was used to identify associations between sociodemographic variables and condom use. Results: most participants were young, male adults, married and with little education. Estimated prevalence of condom use was $23.5 \%$ (95\% Cl; $19.2 \%-27.8 \%$ ). Individuals under 39 years old were found to be 1.82 times more likely to use condoms than individuals aged 40 years or older (OR $=1.82 ; 95 \%$ $\mathrm{Cl})$, while being married reduced the likelihood $(\mathrm{OR}=0.26)$. Conclusion: prevalence of condom use is low among construction workers. Combined prevention is one option for controlling communicable diseases, with condoms as the main support. Descriptors: Construction Industry; Occupational Health; Condoms; Prevalence; Nursing.

\section{RESUMEN}

Objetivo: estimar la prevalencia del uso de condón y los factores asociados en trabajadores de la construcción. Método: en este estudio transversal y analítico con 370 trabajadores del área metropolitana de João Pessoa, Paraíba, Brazil, la variable de resultado fue el uso de condón en la última relación sexual. Se utilizó un cuestionario estructurado y análisis de regresión logística bivariada y múltiple para identificar asociaciones entre las variables sociodemográficas y el uso del condón. Resultados: la mayoría de los participantes fueron jóvenes, varones adultos, casados y con poca educación. La prevalencia estimada del uso de condones fue del 23,5\% (IC del 95\%; 19,2\% - 27,8\%). Se encontró que las personas menores de 39 años tenían 1,82 veces más probabilidades de usar condones que las personas de 40 años o más (OR =1,82; IC del 95\%), mientras que estar casado redujo la probabilidad $(O R=0,26)$. Conclusión: la prevalencia del uso de condones es baja entre los trabajadores de la construcción. La prevención combinada es una opción para controlar las enfermedades transmisibles, con el condón como principal apoyo.

Descriptores: Industria de la Construcción; Salud Laboral; Condones; Prevalência; Enfermería.

\section{INTRODUÇÃO}

O uso do preservativo tem evitado cerca de 50 milhões de novas infecções pelo HIV desde o início da epidemia. A relação sexual desprotegida é um fator de risco significativo à difusão das Infecções Sexualmente Transmissíveis (ISTs) e, principalmente, do HIV em todo o mundo. Esse fator, por si só, já torna o uso do preservativo como um componente imprescindível à diminuição dos ciclos de contaminações ${ }^{1}$.

'Enfermeira. Mestranda pelo Programa de Pós Graduação em Enfermagem da Universidade Federal da Paraíba. João Pessoa, Paraíba, Brasil. E-mail: layanetrindades@hotmail.com. ORCID: https://orcid.org/0000-0001-7124-2187

"Enfermeira. Mestranda pelo Programa de Pós Graduação em Enfermagem da Universidade Federal da Paraíba. João Pessoa, Paraíba, Brasil. E-mail: aparecidacatao@gmail.com. ORCID: https://orcid.org/0000-0002-0017-4209

"'Doutora. Professora Adjunta IV pela Universidade Federal da Paraíba. João Pessoa, Paraíba, Brasil. E-mail: enf.elimoreirafreire@gmail.com. ORCID: https://orcid.org/0000-0002-0305-4843 IVEnfermeira. Mestre pelo Programa de Pós-Graduação em Enfermagem da Universidade Federal da Paraíba. João Pessoa, Paraíba, Brasil. E-mail: wynnenogueira@hotmail.com. ORCID: https://orcid.org/0000-0002-7492-7939

vEnfermeira. Mestranda pelo Programa de Pós Graduação em Enfermagem da Universidade Federal da Paraíba. João Pessoa, Paraíba, Brasil. E-mail: cibellynf@hotmail.com. ORCID: https://orcid.org/0000-0003-4139-0036

VIDoutora. Professora Adjunta da Universidade Federal da Paraíba. João Pessoa, Paraíba, Brasil. E-mail: anacris.os@gmail.com. ORCID: https://orcid.org/0000-0001-8605-5229 
No Brasil, o uso do preservativo diminuiu, principalmente, entre os mais jovens, na faixa etária de 15 a 24 anos, tanto com parceiros eventuais como com parceiros fixos ${ }^{2}$. O hábito de não usar o método de barreira tem impacto direto no aumento de casos de HIV. No país, 52,7\% dos casos de HIV ocorrem na faixa etária de 20 a 34 anos ${ }^{3}$. Somente em 2018, estima-se que ocorreram 100.000 novas infecções e uma em cada cinco são jovens entre 15 e 24 anos ${ }^{4}$. Na América Latina, tais infecções entre os jovens representam $20 \%$ de todas as novas infecções 5 .

Desse modo, pensando nas possibilidades que oportunizem diminuir a prevalência das ISTs, os países, em todo o mundo, têm se esforçado no sentido de combater a propagação dessas infecções. O preservativo tem sido, assim, a forma de prevenção mais divulgada pelas políticas de saúde por seu elevado grau de proteção frente ao risco do aparecimento das ISTs, sobretudo, o HIV e por, efetivamente, reduzir os ciclos de contaminações ${ }^{6}$.

No entanto, o acesso da população aos métodos de prevenção aqui destacados, principalmente aqueles vulneráveis às ISTs, ainda é um desafio enfrentado, emergindo esforços e atenção das diversas esferas da saúde. Tornase imperativo que os órgãos municipais responsáveis por essa pasta busquem estratégias efetivas para que os preservativos estejam disponíveis onde a população mais vulnerável se encontra, a exemplo daqueles que fazem parte das populações-chave e das populações prioritárias, bem como em escolas e instituições sociais e organizações não governamentais ${ }^{7}$.

A diversidade do trabalho de construção civil dificulta a padronização ou a prática de intervenções de prevenção e promoção da saúde, que sejam significativas, nos canteiros de obras ${ }^{8,9}$. Quanto a estabelecimentos da construção civil em último levantamento (2016), o Nordeste apresentava 35.923 estabelecimentos. Destes, 3.914 localizava-se na Paraíba e 2.153 estavam situados precisamente na capital João Pessoa ${ }^{10}$.

Trata-se de uma profissão predominantemente masculina e, nesse contexto a presença da população masculina na Atenção Primária à Saúde (APS) é menos efetiva, atingindo, até mesmo, índices de invisibilidade quando analisados sob as perspectivas de gênero ${ }^{11}$. Reconhecer esta população como propensa a não utilização desse método de barreira e conhecer os principais fatores associados a essa suscetibilidade são fundamentais ao direcionamento das ações desempenhadas pelos profissionais da saúde do trabalhador.

No universo dos trabalhadores da construção civil, o uso inconsistente do preservativo vem sendo debatido na literatura, porém poucos estudos mostram uma boa adesão ao método por essa população específica ${ }^{12,13}$. São, em sua maioria, jovens, sexualmente ativos, de baixa escolaridade, submetidos a processos migratórios constantes. Tais fatores favorecem os comportamentos de risco, como consumo de álcool e drogas, multiplicidade de parceiros e baixa adesão ao uso de preservativos, tornando-os vulneráveis às ISTs, incluindo o HIV ${ }^{13,14}$.

Refletir sobre o uso do preservativo pelos trabalhadores atuantes na Indústria da Construção Civil (ICC) torna-se indiscutível. Das 91.861 milhões de pessoas com idade de 14 anos ou mais ocupadas, no Brasil, cerca de 6,6 milhões tiveram emprego vinculado à ICC até dezembro de $2018^{15}$.

É essencial que o enfermeiro do trabalho, em parceria com a rede social desses indivíduos, busque, de forma combinada e com intervenções contínuas e duradouras, promover uma discussão que garanta uma assistência integral, igualitária e acessível no contexto de comportamento e práticas sexuais ${ }^{16}$.

Nesse contexto, identificar o uso do preservativo em trabalhadores da construção civil configura-se como uma estratégia importante para a interrupção da cadeia de transmissão dessas infecções, particularmente entre aqueles que, potencialmente, têm menos acesso aos serviços de saúde. Ademais, analisar as causas que induzem os trabalhadores a tomar práticas sexuais seguras, sua motivação para o uso de preservativos e suas preocupações com a saúde sexual são de suma relevância para a agenda da saúde pública no país.

O objetivo deste estudo é, portanto, identificar a prevalência do uso do preservativo na última relação sexual e os fatores associados, em trabalhadores da construção civil, no município de João Pessoa, no estado da Paraíba.

\section{METOdologia}

Estudo transversal, analítico, realizado com trabalhadores da construção civil do município de João Pessoa e regiões metropolitanas (Bayeux, Santa Rita e Cabedelo), no estado da Paraíba, realizado no período de março a setembro de 2018

Para o cálculo de tamanho amostral foi considerada uma população estimada de 5.500 trabalhadores, uma estimativa de prevalência de $50 \%$, recomendado quando não se sabe previamente a prevalência real de se ter ou não a condição desejada a investigar, estimando-a então como aleatória ${ }^{17}$. Ainda com nível de confiança de $95 \%$ e margem de erro de 5\%, resultando num tamanho mínimo da amostra de 360 participantes.

Foram incluídos indivíduos com idade igual ou superior a 18 anos, atuando na função no momento da pesquisa. $\mathrm{E}$ foram excluídos do estudo indivíduos que não iniciaram vida sexual ou não tiveram relação sexual nos últimos 12 meses. 
Ao final do estudo, a amostra mínima foi extrapolada e composta por 370 trabalhadores. 0 processo de amostragem foi do tipo não probabilística, obtida por conveniência.

A coleta de dados aconteceu nas próprias unidades de construção, por meio da articulação com o Técnico de Segurança do Trabalho, o qual agendou data e horário para os encontros dos pesquisadores e participantes. Foram visitadas oito unidades de construção, todas de condomínio vertical.

O recrutamento dos indivíduos foi feito durante períodos de descanso, de forma sequencial e de acordo com a chegada do trabalhador no local de descanso. Todos os trabalhadores elegíveis foram convidados a participar do estudo e informados sobre sua importância, objetivos, riscos e benefícios da participação, assim como a liberdade de não participar da pesquisa a qualquer momento. Para indivíduos que participaram, foi oferecido o termo de consentimento livre e esclarecido (TCLE) para leitura e assinatura. Nos casos de analfabetismo, o TCLE foi lido para o funcionário e uma testemunha, e a assinatura do participante foi dactiloscópica.

As informações foram colhidas por meio de um questionário estruturado adaptado do instrumento utilizado na Pesquisa de Conhecimentos, Atitudes e Práticas na População Brasileira² ${ }^{2}$ contendo dados sociodemográficos e possíveis fatores associados aos comportamentos de risco para as ISTs.

Para a análise dos dados, considerou-se o uso do preservativo masculino ou feminino na última relação sexual, como variável de desfecho, pois ambos se mostram relevantes para a prevenção de ISTs ${ }^{18}$. Quanto à escolha da variável desfecho, existem diferentes medidas para se avaliar o uso do preservativo, cada uma delas apresentando vantagens e desvantagens. No entanto, um dos indicadores utilizados em relatórios globais, para medir a prevalência do uso de preservativos, é o uso de preservativos na última relação sexual ${ }^{19}$.

As variáveis categóricas foram descritas em números absolutos e percentuais. As variáveis sociodemográficas e comportamentais: idade (18 a 39 anos, acima de 39 anos); estado civil (casado, solteiro/outros); número de parceiros (um parceiro, dois ou mais parceiros); tipo de parceiro (sexo oposto, qualquer sexo); uso de álcool (sim, não); e uso de drogas ilícitas (sim, não), foram utilizadas no sentido de investigar quais são os fatores de risco e de proteção para o uso do preservativo. Para tanto, foi aplicada a análise bivariada do modelo de regressão logística, gerando as razões de chances (RC) ou odds ratio (OR) com intervalo de confiança de $95 \%$. As variáveis estatisticamente significantes $(p<0,05)$ foram incluídas simultaneamente no modelo de regressão logística múltipla, com teste de verossimilhança.

A pesquisa obteve aprovação pelo Comitê de Ética e Pesquisa da instituição (Parecer no 2.572.58) e respeitou todos os preceitos éticos da pesquisa que envolve seres humanos ${ }^{20}$

\section{RESULTADOS}

Quanto à prevalência de uso do preservativo, observou-se que, do total de 370 indivíduos pesquisados, 87 disseram ter usado preservativo na última relação sexual, o que representa uma prevalência de 23,5\% (IC 95\%: $19,2 \%$ $27,8 \%)$ ou, aproximadamente, 24 indivíduos a cada 100 trabalhadores da construção civil fizeram uso do preservativo na última relação.

Quanto às características sociodemográficas, verificou-se o predomínio de trabalhadores do sexo masculino, 361 (97,6\%); entre 30 e 39 anos de idade, 127 (34,3\%); entre cinco e oito anos de estudo, 125 (33,8\%). Quanto ao estado civil, a maioria era casado(a) ou em união consensual, 309 (83,5\%). Quanto à renda mensal, 220 (59,5\%) trabalhadores recebiam entre um a 1,5 salários mínimos, com valor referente de $\mathrm{R} \$ 954,00$. A maioria era procedente do interior do estado da Paraíba, 161 (43,5\%), e 131 (35,4\%) tinham tempo de atuação na ICC de seis a 10 anos.

Os dados relacionados à prática sexual e ao uso do preservativo por trabalhadores de construção civil estão dispostos na Tabela 1.

Destaca-se que quanto ao conhecimento do preservativo masculino e feminino, 365 (98,6\%) e 231 (62,4\%) relataram conhecê-lo, respectivamente. Quando questionados sobre a utilização do preservativo na última relação, 283 $(76,5 \%)$ afirmaram não ter feito uso. Com relação à frequência do uso do preservativo nos últimos 12 meses, 181 (48,9\%) responderam que nunca o utilizaram e $130(35,1 \%)$ disseram que usaram o preservativo às vezes.

Os dados relacionados ao comportamento sexual de risco e ao uso do preservativo por trabalhadores de construção civil estão dispostos na Tabela 2.

Quanto ao número de parceiros sexuais nos últimos 12 meses, 259 (70,0\%) referiram ter um único parceiro, seguido de dois ou mais, 111 (30,0\%). A maioria dos trabalhadores, $366(98,9 \%)$, relatou prática de atividade sexual com pessoas do sexo oposto.

Diante da afirmação que o uso de álcool ou drogas pode induzir à relação sexual sem camisinha, 323 (87,3\%) concordam com a assertiva. Observa-se que a maior parte, 254 (68,6\%), referiu não ter relação sexual com profissionais 
do sexo (Tabela 2). Ao serem indagados se haviam recebido dinheiro ou efetuado pagamento em troca de sexo, 97 $(26,2 \%)$ dos entrevistados responderam que sim e destes, 80 (82,5\%) afirmaram ter usado preservativo.

TABELA 1: Conhecimento, atitudes e prática do uso do preservativo de trabalhadores da construção civil no município de João Pessoa e Região Metropolitana ( $n=370)$. João Pessoa, PB, Brasil, 2018.

\begin{tabular}{lll}
\hline Variável & $\mathbf{n}$ & $\mathbf{\%}$ \\
\hline Conhece o preservativo masculino? & 365 & 98,6 \\
Sim & 5 & 1,4 \\
Não & & \\
Conhece o preservativo feminino? & 231 & 62,4 \\
Sim & 139 & 37,6 \\
Não & & \\
Pensando na última relação sexual, você usou o preservativo? & 87 & 23,5 \\
Sim & 283 & 76,5 \\
Não & & \\
Frequência do uso do preservativo nos últimos 12 meses? & 59 & 16,0 \\
Sempre & 130 & 35,1 \\
Às vezes & 181 & 48,9 \\
Nunca & 370 & $\mathbf{1 0 0 , 0}$ \\
\hline TOTAL & & \\
\hline
\end{tabular}

Fonte: Dados da pesquisa, 2018

TABELA 2: Comportamento sexual de risco e uso do preservativo de trabalhadores da construção civil no município de João Pessoa e Região Metropolitana ( $n=370)$. João Pessoa, PB, Brasil, 2018.

\begin{tabular}{|c|c|c|}
\hline Variável & $\mathbf{n}$ & $\%$ \\
\hline \multicolumn{3}{|c|}{ Número de parceiros(as) sexuais nos últimos 12 meses? } \\
\hline 1 parceiro & 259 & 70,0 \\
\hline$\geq 2$ parceiros & 111 & 30,0 \\
\hline \multicolumn{3}{|c|}{ Tipo de parceiros(as) sexuais nos últimos 12 meses? } \\
\hline Sexo oposto & 366 & 98,9 \\
\hline Qualquer sexo & 4 & 1,1 \\
\hline \multicolumn{3}{|c|}{$\begin{array}{l}\text { Concorda com a seguinte afirmação: "o uso de álcool ou drogas pode fazer com } \\
\text { que as pessoas tenham relação sexual sem usar camisinha" }\end{array}$} \\
\hline Sim & 323 & 87,3 \\
\hline Não & 47 & 12,7 \\
\hline \multicolumn{3}{|c|}{ Teve relação sexual com profissional do sexo? } \\
\hline Sim & 116 & 31,4 \\
\hline Não & 254 & 68,6 \\
\hline \multicolumn{3}{|c|}{ Já teve relação sexual com alguém que conheceu pela internet? } \\
\hline Sim & 26 & 7,0 \\
\hline Não & 344 & 93,0 \\
\hline \multicolumn{3}{|c|}{ Já fez sexo com parceiro sexual que conheceu pelo celular (Tinder, Badoo)? } \\
\hline Sim & 32 & 8,6 \\
\hline Não & 338 & 91,4 \\
\hline TOTAL & 370 & 100,0 \\
\hline
\end{tabular}

Fonte: Dados da pesquisa, 2018.

Quanto aos aplicativos móveis, $344(93,0 \%)$ trabalhadores não tiveram relação com alguém que conheceram pela internet, bem como $338(91,4 \%)$ trabalhadores nunca fizeram sexo com parceiro que conheceram pelo celular (Tinder, Badoo, etc), conforme disposto na Tabela 2. Dentre os 32 (8,6\%) indivíduos que fizeram sexo com esses parceiros conhecidos por essas vias, $18(56,3 \%)$ afirmaram que sempre usaram o preservativo. 
Os dados relacionados à associação entre os fatores de risco e o uso do preservativo por trabalhadores de construção civil estão dispostos na Tabela 3. É importante destacar que, para todas as análises em que foram consideradas medidas de risco relativo (odds ratio), a última categoria de cada uma das variáveis categóricas analisadas foi considerada de referência.

TABELA 3: Associação entre ascaracterísticas sociodemográficas e comportamentais de trabalhadores da construção civil em relação ao uso de preservativo na última relação sexual, no município de João Pessoa e Região Metropolitana ( $n=370)$. João Pessoa, PB, Brasil, 2018.

\begin{tabular}{|c|c|c|c|c|c|}
\hline Variáveis & $\begin{array}{c}\text { Usou } \\
\text { preservativo } \\
\mathrm{n}(\%) \\
\end{array}$ & $\begin{array}{c}\text { Não usou } \\
\text { preservativo } \\
\mathrm{n}(\%) \\
\end{array}$ & $\begin{array}{c}\text { Razão de } \\
\text { chances } \\
\text { (RC) }\end{array}$ & IC 95\% & $p$-valor \\
\hline \multicolumn{6}{|l|}{ Idade } \\
\hline 18 a 39 anos & $59(67,8)$ & $152(53,7)$ & 1,82 & $1,10-3,01$ & $0,03^{(*)}$ \\
\hline$\geq 40$ anos & $28(32,2)$ & $131(46,3)$ & 1 & & \\
\hline \multicolumn{6}{|l|}{ Estado civil } \\
\hline Casado & $58(18,8)$ & $251(47,5)$ & 0,26 & $0,14-0,45$ & $0,001^{(*)}$ \\
\hline Solteiro/outros & $29(81,2)$ & $32(52,5)$ & 1 & & \\
\hline \multicolumn{6}{|c|}{ Número de parceiros } \\
\hline 1 Parceiro & $58(66,7)$ & $201(71,0)$ & 0,82 & $0,49-1,36$ & 0,52 \\
\hline$\geq 2$ Parceiros & $29(33,3)$ & $82(29,0)$ & 1 & & \\
\hline \multicolumn{6}{|l|}{ Tipo de parceiro } \\
\hline Sexo oposto & $85(97,7)$ & $281(99,3)$ & 0,30 & $0,04-2,18$ & 0,51 \\
\hline Qualquer sexo & $2(2,3)$ & $2(0,7)$ & 1 & & \\
\hline \multicolumn{6}{|l|}{ Uso de álcool } \\
\hline Sim & $58(66,7)$ & $176(62,2)$ & 1,22 & $0,73-2,02$ & 0,53 \\
\hline Não & $29(33,3)$ & $107(37,8)$ & 1 & & \\
\hline \multicolumn{6}{|c|}{ Uso de drogas ilícitas } \\
\hline $\operatorname{Sim}$ & $11(12,6)$ & $47(16,6)$ & 0,73 & $0,36-1,47$ & 0,47 \\
\hline Não & $76(87,4)$ & $236(83,4)$ & 1 & & \\
\hline
\end{tabular}

Fonte: Dados da pesquisa, 2018. Intervalo de Confiança (IC). 1- categoria de referência. ${ }^{(*)}$ Resultado significativo com $\mathrm{p}<0,05$.

Quanto ao uso ou não de preservativo na última relação sexual pelos trabalhadores da ICC e a associação com as características sociodemográficas, observou-se associação significativa para a idade e o estado civil. As variáveis idade e o estado civil dos participantes são significativas na análise do desfecho, que é o uso do preservativo na última relação sexual. Além disso, trabalhadores com idade entre 18 e 39 anos possuem 1,82 vezes mais chances de usar o preservativo quando comparados com os indivíduos com idade maior ou igual a 40 anos, sendo este aumento de no máximo 3,01, com base no nível de confiança considerado e no limite superior do intervalo de confiança construído.

Quanto ao estado civil, ser casado pode diminuir em até 3,84 vezes as chances de uso do preservativo quando comparado à categoria ser solteiro $(R C=1 / 0,26)$, e essa diminuição pode ser de até 7,14 vezes. Para as demais variáveis, não foram encontradas evidências de aumento ou diminuição das chances de uso do preservativo entre as categorias.

Realizou-se análise de regressão logística múltipla para medir qual a influência desses fatores conjuntamente. A equação do modelo de regressão logística que estima o valor da variável dependente "uso de preservativo" com resultado significativo, medido através do qui-quadrado para o teste de verossimilhança $\left(\chi^{2}=73,17 ; g l=2 ; p<0,001\right)$, em relação às variáveis independentes e preditoras "idade e o estado civil" foi: Logit Pi $=-0,13+(10,26 \mathrm{x}$ idade $)-(11,09 \mathrm{x}$ estado civil). Muito embora, quando analisados conjuntamente, esses dois fatores (um aumentando a chance e o outro diminuindo a chance) parecem se anularem, resultando num Odds com intervalo de confiança de zero a infinito e $p$ valores não significativos ( $p>0$ 05).

\section{DISCUSSÃO}

Muitos fatores e comportamentos contribuem para a disseminação das ISTs, incluindo a reduzida adesão ao uso do preservativo, baixas condições socioeconômicas e populações vulneráveis, a exemplo de caminhoneiros e populações migrantes, bem como trabalhadores da construção civil ${ }^{12}$. Diante da escassez de estudos que abordem o 
uso do preservativo por trabalhadores da construção civil, os resultados desta investigação serão discutidos com base nos achados de outras pesquisas envolvendo populações consideradas vulneráveis.

Observa-se, neste estudo, que a maioria dos entrevistados possui baixa escolaridade e baixa renda familiar. Estudos apontam associação entre o não uso de preservativos com condições socioeconômicas baixas assim como associação positiva entre status socioeconômico e uso de preservativo entre adolescentes do sexo masculino ${ }^{21,22}$. Diante disso, variáveis socioeconômicas representam uma importante característica epidemiológica que pode influenciar na adoção do preservativo como método de barreira para a prática sexual segura.

A prevalência do uso do preservativo na última relação sexual, identificada nesse estudo, foi baixa, 87 (23,5\%) (IC 95\%: $19,2 \%$ - 27.8\%), o que corrobora os resultados identificados em outras pesquisas envolvendo populações vulneráveis ${ }^{23,24}$. A maioria dos trabalhadores da ICC não usou preservativo na última relação sexual.

O fato de sentir-se ou não susceptível pode ser o motivador do uso ou não uso do preservativo. Ademais, a adesão consistente pode ter relação com a possibilidade ou não de estar em risco de contrair alguma infeç̧ão ${ }^{25}$. Além disso, a influência do uso de álcool e drogas, a confiança no(a) parceiro(a), a masculinidade ${ }^{26}$ e o desconhecimento do método de barreira podem ser fatores que também interferem no uso do preservativo ${ }^{27}$. Nesse contexto, é importante a elaboração de propostas de intervenções efetivas para incentivar o uso de preservativos por populações vulneráveis, a exemplo de trabalhadores de construção civil.

No presente estudo, quanto maior a idade, menor é a adesão ao uso do preservativo. Esse tipo de comportamento é observado em pessoas mais velhas, as quais tendem a manter relações sexuais sem preservativo, principalmente nas relações estáveis ${ }^{28}$.

Um tópico que chama atenção e que reflete as formas de relacionamento nos tempos modernos com os adventos tecnológicos é o resultado quanto ao recrutamento de parceiros por aplicativos móveis e o fato de não usar preservativo na relação. A exemplo disso, inquérito brasileiro relatou associação entre maior rotatividade de parceiros sexuais encontrados por aplicativos de encontro ${ }^{29}$.

Quanto ao estado civil, verificou-se que os trabalhadores que são casados têm uma menor chance de usar o preservativo. As diferentes relações estabelecidas, estáveis ou não estáveis, podem influenciar a adoção do uso de preservativos $^{30}$. Existe maior dificuldade em manter o uso do preservativo em relações estáveis ${ }^{25}$. Relações estáveis, para a maioria das pessoas, representam ausência de risco para contrair alguma IST e, por isso, negligenciam o uso do preservativo ${ }^{31-33}$, tornando-se um desafio para traçar estratégias de prevenção, principalmente, relacionadas ao controle das ISTs,

Estudo desenvolvido com caminhoneiros apontou a baixa adesão ao uso do preservativo com suas parceiras fixas. Muitas vezes, a relação estável representa fatores interligados à confiança, ao cuidado, à estabilidade das relações pessoais e à idealização do relacionamento como forma de prevenção para as diferentes infecções ${ }^{34}$. Considerando esse panorama, os indivíduos tornam-se mais vulneráveis ao HIV e outras ISTs do que aqueles que continuam adotando o método para a prevenção das ISTs ${ }^{35}$.

Os dados mencionados acima são corroborados por um estudo desenvolvido com migrantes internacionais, o qual identificou que esposas de trabalhadores migrantes, frequentemente, acreditam que seus maridos são confiáveis para protegê-las do HIV e que esses não possuem relação extraconjugal ${ }^{36}$. No entanto, estudo desenvolvido em Moscou com trabalhadores migrantes mostrou que ter parceira regular está associado a ter relações extraconjugais sem uso do preservativo $^{37}$.

Partindo desse pressuposto, a escuta ativa e a promoção de um ambiente favorável ao diálogo sobre as práticas sexuais são imperativas nos serviços de saúde, especialmente nos serviços de saúde do trabalhador. Essa abordagem possibilita vínculos e facilita a adesão às biotecnologias disponíveis ${ }^{7}$.

Não obstante, o uso do preservativo deve ser avaliado de forma ampla e contextualizada, tendo em vista que as pessoas poderiam referir não ter usado preservativo em determinada relação sexual, mas nem por isso ter-se exposto a alguma IST, em função de outras modalidades de prevenção terem sido adotadas. Deve-se pensar na adoção de uma combinação de estratégias de prevenção, que inclua: o uso do preservativo e o julgamento do quanto esse é considerado o método mais adequado ${ }^{30}$.

As condições de trabalho e vida dos trabalhadores da ICC tendem a amplificar algumas condutas masculinas associadas ao maior risco sexual de contrair alguma IST como múltiplas parceiras sexuais, o consumo de bebidas alcóolicas e ter relação sexual com profissionais do sexo sem preservativo. Mudar essas condições ou fortalecer a capacidade dos trabalhadores, alojados em casas de apoio, além de encontrar alternativas para lidar com essas dificuldades também podem ser o foco de prevenção na saúde do trabalhador. Por exemplo, existem outras vias de socialização, que não sejam o álcool e o lazer sexual ${ }^{36}$. 
Desse modo, pensando na saúde do trabalhador, o enfermeiro é essencial em qualquer empresa com trabalhadores. O ambiente de trabalho oferece variados riscos à saúde dos indivíduos, que podem ser evitados ou reduzidos por meio de medidas de proteção variadas. No contexto das ISTs, percebe-se que por desconhecer ou não identificar determinadas situações de risco, o trabalhador encontra-se mais vulnerável ${ }^{37}$.

A enfermagem do trabalho, além de atuar nas doenças ocupacionais e relacionadas ao trabalho, precisa ter um olhar voltado a outras doenças e agravos não ocupacionais e que são transversais à saúde do trabalhador. As ações de promoção da saúde em enfermagem do trabalho contribuem para o empoderamento dos trabalhadores sobre seu próprio processo saúde-doença, com vistas à construção de um ambiente de trabalho produtivo e seguro que satisfaça as necessidades econômicas e individuais do trabalhador ${ }^{37}$.

A pesquisa apresenta limitações que devem ser pontuadas. A primeira relacionada ao desenho do estudo, uma vez que estudos transversais não permitem realizar inferências causais. Outra limitação refere-se à variável desfecho, que pode ter introduzido um viés de informação no estudo, pois os entrevistados podem ter se sentido constrangidos ao responder às perguntas de cunho sexual.

É importante deixar claro que este estudo avaliou o uso do preservativo na última relação sexual e que não mediu seu uso consistente, pois não foram avaliadas a continuidade nem a frequência do uso de preservativos. Para isso, seria necessária a utilização de outras medidas, as quais não foram o objetivo deste estudo. Logo, a prevalência de uso pode estar sendo superestimada, podendo a diferença encontrada ser menor do que a diferença real ou até mesmo não ser identificada uma diferença.

\section{CONCLUSÃO}

Os trabalhadores de construção civil de João Pessoa, sobre o qual este estudo se debruçou, demonstraram baixa adesão ao uso do preservativo. Ser casado também foi associado negativamente. Em relação à idade, constatou-se que quanto menor a idade do indivíduo, maior sua chance de usar preservativo.

Conclui-se, assim, que, independentemente do modelo de prevenção adotado no Brasil, é necessário haver uma regularidade nos estudos nacionais que avaliem o uso do preservativo nos diferentes segmentos populacionais. $A$ política de prevenção deve se basear na combinação de estratégias quanto à adesão ao uso do preservativo e demais métodos de prevenção das ISTs. Ainda se faz necessário que os trabalhadores atuantes na ICC sejam inseridos nas políticas públicas de atenção à saúde sexual voltada ao trabalhador.

Ademais, a oferta de preservativo deve acontecer nos ambientes de trabalho. Os preservativos devem estar disponíveis sem barreiras, 24 horas por dia, inclusive aos finais de semana e feriados, nas casas de apoio desses trabalhadores e no serviço especializado de saúde do trabalhador das empresas, de modo que a distribuição de preservativo para os trabalhadores da ICC concretize a política de prevenção no Brasil através da livre oferta dos preservativos.

Ressalta-se que a prevenção combinada remete à conjunção de diferentes ações na prevenção às ISTs. O melhor método de prevenção deve ser adequado à realidade e ao contexto de cada indivíduo. No entanto, o uso do preservativo continua sendo o principal coadjuvante na prevenção combinada tanto pela disponibilidade e facilidade em adquiri-lo quanto pela sua eficácia. Afinal, quem tem um preservativo poderá usá-lo ou não, mas quem não o tem certamente não o usará.

Espera-se que este estudo contribua para a reflexão desta temática e que estimule o desenvolvimento de novas pesquisas que permitam uma investigação mais ampla para acrescentar dados às pesquisas já existentes, considerando a importância da saúde desses trabalhadores e o desafio de ações eficientes para o combate das ISTs para novos modelos de assistência e, assim, implantar medidas que amenizem os fatores que desencadeiam a não adoção do uso preservativo.

\section{REFERÊNCIAS}

1. World Health Organization (WHO). Consolidated guidelines on HIV prevention, diagnosis, treatment and care for key populations. [Internet]. 2014 [cited 2019 Mar 07]. Available from: http://www.paho.org/bra/images/stories/Documentos2/eng\%20guias\%20pop\%20vul\%20who-1.pdf?ua=1

2. Ministério da Saúde (BR). Secretária de Vigilância em Saúde. Departamento de DST, Aids e Hepatites Virais. Pesquisa de conhecimentos, atitudes e práticas na população brasileira. Brasília: MS; 2011. [cited 2020 Apr 07]. Available from: http://bvsms.saude.gov.br/bvs/publicacoes/pesquisa_conhecimentos_atitudes_praticas_populacao_brasileira.pdf

3. Ministério da Saúde (BR). Boletim Epidemiológico - HIV/Aids. Secretaria de Vigilância em Saúde. [Internet] 2019 [cited 2020 Mar 31]. Available from: http://www.aids.gov.br/pt-br/pub/2019/boletim-epidemiologico-de-hivaids-2019 
4. Programa das Nações Unidas sobre HIV/aids (UNAIDS). Campanha que incentiva diálogo aberto sobre HIV e prevenção entre profissionais de saúde e jovens. [Internet]. 2019 [cited 2020 Mar 31]. Available from: https://unaids.org.br/2019/12/unaids-eparceiros-lancam-campanha-que-incentiva-dialogo-aberto-sobre-hiv-e-prevencao-entre-profissionais-de-saude-e-jovens/

5. World Health Organization (WHO). Status of HIV epidemic and response. [Internet]. 2019 [cited 2020 Mar 31]. Available from: https://www.paho.org/hq/index.php?option=com_docman\&view=download\&slug=status-of-hiv-epidemic-and-response-latinamerica-and-the-caribbean-2019\&ltemid=270\&lang=en

6. Ministério da Saúde (BR). Boletim Epidemiológico - HIV/Aids. Secretaria de Vigilância em Saúde. [Internet]. 2018 [cited 2019 Jun 18]. Available from: http://www.aids.gov.br/pt-br/pub/2018/boletim-epidemiologico-hivaids-2018

7. Ministério da Saúde (BR). Departamento de Doenças de Condições Crônicas e Infecções Sexualmente Transmissíveis. Protocolo Clínico e Diretrizes Terapêuticas para Atenção Integral às Pessoas com Infecções Sexualmente Transmissíveis (IST). [Internet] 2020 [cited 2019 Jun 22]. Available from: http://www.aids.gov.br/system/tdf/pub/2016/57800/pcdt_ist_final_revisado_020420.pdf?file=1\&type=node\&id=57800\&force=1

8. Meintjes I, Bowen P, Root D. HIV/AIDS in the South African construction industry: understanding the HIV/AIDS discourse for a sector specific response. Construct Manag Econ. [Internet]. 2007 [cited 2019 Jun 18]; 25(3): 255-66. DOI: https://doi.org/10.1080/01446190601071813

9. Bowen P, Govender R, Edwards P, Cattell K. HIV testing of construction workers in the Western Cape, South Africa. AIDS care. [Internet]. 2015 [cited 2019 Jun 18]; 27(9): 1150-55. Available from: https://pubmed.ncbi.nlm.nih.gov/25886973/

10. Câmara Brasileira de Indústria da Construção (CBIC). Estabelecimentos na Construção. Número de estabelecimentos na construção civil. [Internet]. 2017 [cited 2019 Aug 05]. Available from: http://www.cbicdados.com.br/menu/empresas-deconstrucao/estabelecimentos-na-construcao

11. Couto MT, Pinheiro TF, Valença O, Machin R, Silva GSN, Gomes R. et al. Men in primary healthcare: discussing (in) visibility based on gender perspectives. Interface (Botucatu) [Internet]. 2010 [cited 2019 Jun 09]; 14(33):257-70. DOI: https://doi.org/10.1590/S1414-32832010000200003

12. Tarkang EE, Pencille LB. Psychosocial predictor of consistent condom use among migrant road construction workers in the Southwest Region of Cameroon using the Health Belief Model. Pan Afr Med J. [Internet]. 2018 [cited 2019 Jun 20]; 29:215. Available from: https://www.ncbi.nlm.nih.gov/pmc/articles/PMC6080963/

13. Bowen $P$, Govender R, Edwards P. Validating survey measurement scales for AIDS-related knowledge and stigma among construction workers in South Africa. BMC Public Health. [Internet]. 2016 [cited 2019 Jun 18]; 16(1):70. Available from: https://pubmed.ncbi.nlm.nih.gov/26803294/

14. Sadarangani SP, Lim PL, Vasoo S. Infectious diseases and migrant worker health in Singapore: a receiving country's perspective. J Travel Med. [Internet]. 2017 [cited 2019 Jun 18]; 24(4). DOI: https://doi.org/10.1093/jtm/tax014

15. Câmara Brasileira de Indústria da Construção (CBIC). Estabelecimentos na Construção. Valor adicionado bruto - Construção civil. [Internet]. 2018 [cited 2019 Aug 05]. Available from: http://www.cbicdados.com.br/menu/pib-e-investimento/pib-brasil-econstrucao-civil

16. Carvalho PMRS, Guimarães RA, Moraes PA, Teles SA, Matos MA. Prevalence of signs and symptoms and knowledge about sexually transmitted diseases. Acta Paul. Enferm. (Online). 2015 [cited 2019 Aug 05]; 28(1):95-100. DOI: https://doi.org/10.1590/1982-0194201500016

17. Cochran WG. Sampling techniques. John Wiley \& Sons, Hoboken, 2007.

18. Holmes KK, Levine R, Weaver M. Effectiveness of condoms in preventing sexually transmitted infections. Bull World Health Organ. [Internet]. 2004 [cited 2020 Mar 30]; 82(6):454-61. Available from:

https://www.scielosp.org/article/bwho/2004.v82n6/454-461/en/

19. Joint United Nations Programme on HIV/AIDS (UNAIDS). Global AIDS response progress reporting 2015. [Internet]. 2015 [cited 2020 Apr 01]. Available from: https://www.unaids.org/sites/default/files/media_asset/JC2702_GARPR2015guidelines_en.pdf

20. Ministério da Saúde (BR). Conselho Nacional de Saúde. Resolução n. 466/12. Dispõe sobre diretrizes e normas regulamentadoras de pesquisas envolvendo seres humanos. Brasília (DF): CNS; 2012. [cited 2019 Aug 07]. Available from: https://bvsms.saude.gov.br/bvs/saudelegis/cns/2013/res0466_12_12_2012.html

21. Martins LB, Costa-Paiva LH, Osis MJ, Sousa MH, Pinto-Neto AM, Tadini V. Factors associated with condom use and knowledge about STD/AIDS among teenagers in public and private schools in Sao Paulo, Brazil. Cad. Saúde Pública (Online). 2006 [cited 2019 Aug 07]; 22(2):315-23. DOI: https://doi.org/10.1590/s0102-311×2006000200009

22. Juarez F, Legrand T. Factors influencing boys' age at first intercourse and condom use in the Shantytowns of Recife, Brazil. Stud Fam Plann. [Internet]. 2015 [cited 2020 Apr 01]; 36(1):57-70. DOI: https://doi.org/10.1111/j.1728-4465.2005.00041.x

23. Castillo-Mancilla J, Allshouse A, Collins C, Hastings-Tolsma M, Campbell TB, Ma Whinney S. Differences in sexual risk behavior and HIV/AIDS risk factors among foreign-born and US-born Hispanic women. J Immigr Minor Health. [Internet]. 2012 [cited 2020 Apr 03]; 14(1):89-99. DOI: https://dx.doi.org/10.1007\%2Fs10903-011-9529-7

24. Santos CMA, Oliveira JDS, Lima SVMA, Santos AD, Góes MAO, Sousa LB. Men's knowledge, attitudes and practice regarding sexually transmitted diseases. Cogitare Enferm. [Internet]. 2018 [cited 2020 Apr 03]; 23(1). DOI: http://dx.doi.org/10.5380/ce.v23i1.54101

25. Hsu HT, Wenzel S, Rice E, Gilreath TD, Kurzban S, Unger J. Understanding consistent condom use among homeless men who have sex with women and engage in multiple sexual partnerships: a path analysis. AIDS Behav. [Internet]. 2015 [cited $2020 \mathrm{Apr}$ 03]; 19:1676-88. Available from: https://pubmed.ncbi.nlm.nih.gov/25845531/ 
26. Serra MAAO, Milhomem AB, Oliveira SB, Santos FAAS, Silva RA, Cunha MCSO, et al. Sociodemographic and behavioral factors associated with HIV vulnerability according to sexual orientation. AIDS Res Treat. [Internet]. 2020 [cited 2020 May 23 ]; 20. Available from: http://downloads.hindawi.com/journals/art/2020/5619315.pdf

27. Kanda L, Mash R. Reasons for inconsistent condom use by young adults in Mahalapye, Botswana. Afr J Prm Health Care Fam Med. [Internet]. 2018 [cited 2020 May 23]; 10(1). DOI: https://doi.org/10.4102/phcfm.v10i1.1492

28. Freitas JLG, Pereira PPS, Moreira KFA, Silva AD. Prevalência do não uso de preservativo entre universitários e pós-graduandos de uma universidade pública do Norte do Brasil. Rev. Eletr. Acervo Saúde. [Internet]. 2019 [cited 2020 May 23]; 25. DOI: https://doi.org/10.25248/reas.e751.2019

29. Queiroz AA, Matos MC, Araújo TM, Reis RK, Sousa AF. Sexually transmitted infections and factors associated with condom use in dating app users in Brazil. Acta Paul. Enferm. (Online). 2019 [cited 2020 May 23]; 32(5):546-53. DOI: https://doi.org/10.1590/1982-0194201900076

30. Dourado I, MacCarthy S, Reddy M, Calazans G, Gruskin S. Revisiting the use of condoms in Brazil. Rev. bras. epidemiol. [Internet]. 2015 [cited 2020 Apr 02]; 1:63-88. DOI: https://doi.org/10.1590/1809-4503201500050006

31. Plutarco LW, Meneses GO, Arruda CM, Holanda LC, Santos WS. A influência da confiança no parceiro na decisão do uso da camisinha. Psicol. Saúde doenças. [Internet]. 2019 [cited 2020 Apr 03]; 20(1):220-33. DOI: http://dx.doi.org/10.15309/19psd200118

32. Hearst N, Chen S. Condom promotion for AIDS prevention in the developing world: is it working? Stud Fam Plann. [Internet]. 2004 [cited 2020 Apr 02]; 35(1):39-47. DOI: https://doi.org/10.1111/j.1728-4465.2004.00004.x

33. Magno L, Castellanos MEP. Significados e vulnerabilidade ao HIV/aids entre caminhoneiros de rota longa no Brasil. Rev. saúde pública (Online). 2016 [cited 2020 May 25]; 50:76. Available from: https://www.scielo.br/pdf/rsp/v50/pt_0034-8910-rsp-S151887872016050006185.pdf

34. Moreira LR, Dumith SC, Paludo SS. Condom use in last sexual intercourse among undergraduate students: how many are using them and who are they? Ciênc. Saúde Colet. [Internet]. 2018 [cited 2020 Apr 02]; 23(4):1255-66. DOI: https://doi.org/10.1590/1413-81232018234.16492016

35. Golobof A, Weine S, Bahromov M, Luo J. The roles of labor migrants' wives in HIV/AIDS risk and prevention in Tajikistan. AIDS Care. [Internet]. 2011 [cited 2020 Apr 02]; 23(1):91-7. Available from: https://pubmed.ncbi.nlm.nih.gov/21218281/

36. Weine $S$, Bahromov M, Loue S, Owens L. HIV sexual risk behaviors and multilevel determinants among male labor migrants from Tajikistan. J Immigr Minor Health. [Internet]. 2013 [cited 2020 Apr 03];15(4): 700-10. Available from: https://pubmed.ncbi.nlm.nih.gov/23054537/

37. Pereira JDS, Oliveira AALD, Araújo BPDL, Eloi ADC.The nurses' role with regards to the risks and vulnerabilities faced by civil construction workers. Rev. Pesqui. (Univ. Fed. Estado Rio J., Online). 2019 [cited 2020 May 27]. Available from: http://www.seer.unirio.br/index.php/cuidadofundamental/article/viewFile/6915/pdf_1 